\title{
Pseudomonas aeruginosa Resistance to Various Commonly Used Antibiotics in 27 Hospital Patients \\ Z Veir ${ }^{1}$, M Mance ${ }^{2}$, H Veir ${ }^{3}$, M Franić ${ }^{1}, N$ Beader ${ }^{4}$, R Šalinović ${ }^{5}$
}

\begin{abstract}
Objective: The aim of this study was to use wound swabs to determine antibiotic resistance patterns in Pseudomonas aeruginosa, which has been shown to be highly resistant to many commonly used antibiotics.
\end{abstract}

Methods: This study observed 27 isolates of the gram-negative bacteria Pseudomonas aeruginosa and tested their resistance to various commonly used antibiotics. The strains were isolated from surgical patients of both sexes and different age groups. Patients were hospitalized in various departments (Department of Internal Medicine, Pediatrics, Dermatology, Ear, Nose and Throat, Neurology, Orthopedics, Oncology, and Physical and Rehabilitation medicine) at our hospital.

Results: According to the resistance patterns found, the most effective antibiotic in our sample population was colistin ( $100 \%$ sensitivity), followed by amikacin ( $88 \%$ sensitivity), tobramicin ( $81 \%$ sensitivity). The least effective antibiotics were found to be carbapenems ( $8 \%$ sensitivity) and ciprofloxacin ( $12 \%$ sensitivity).

Conclusion: The isolates of Pseudomonas aeurginosa show a high degree of resistance ( $>50 \%$ resistance) to commonly used antibiotics such as imipenem and meropenem, ciprofloxacin, gentamicin, netilmicin and piperacillin.

Keywords: Antibiotics, antimicrobial resistance, drug resistance, Pseudomonas aeruginosa

From: ${ }^{1}$ Faculty of Medicine Osijek, Car Hadrijan 10E St. Osijek, Croatia. ${ }^{2}$ Department of Plastic, Reconstructive and Aesthetic Surgery, University Hospital Rebro, Kišpatićeva 12, Zagreb, Croatia. ${ }^{3}$ Department of Otolaryngology, Clinical Hospital Merkur, Zajćeva 19, Zagreb, Croatia. ${ }^{4}$ Faculty of Medicine Zagreb, Salata 3b, Zagreb, Croatia. ${ }^{5}$ Premium Dent Clinic, Zagreb, Croatia

Correspondance: Dr M Mance, Department of Plastic, Reconstructive and Esthetic Surgery University Hospital Rebro, Kišpatićeva 12, Zagreb, Croatia. Fax: +385-(0)91-895-3433, e-mail:markomance@gmail.com 


\section{INTRODUCTION}

Gram-negative bacteria such as Pseudomonas Aeruginosa (PA) are significant causes of hospital infections and sepsis. It is often seen in epidemics and represents a therapeutic problem faced by physicians because of the development of resistance to many antibiotics which hinders their eradication (1).

Risk factors for the colonization of a wound or infection that are observed during many epidemics caused by this bacteria include: the presence of an intravascular catheter that can lead to sepsis as a result of the colonization of the skin, emergency abdominal surgery ( gastrointestinal colonization ), length of stay in the hospital or intensive care unit ( mainly the bacteria found in the seriously ill person in the intensive care unit or in the chronically ill patients with a long hospital stay), the severity of the disease, previous stay in an old age home, the presence of urinary catheters, assisted breathing machines and prior therapy with antibiotics (2). Previous literature usually refers to the use of cephalosporins of the III generation, fluoroquinolones, cotrimoxazole, aminoglycosides and penems in order to treat such infections (3)

PA has been shown to possess a variety of antibiotic resistant properties which render this dangerous for the patient and can pose difficulties for the physician since such infections can be difficult to treat, especially in a patient with many co-morbidities and serious illness. PA has shown antibiotic resistance by utilizing many mechanisms. These include efflux pumps, decreased permeability of cellular envelopes, hyper mutation, production of antibiotic inactivating enzymes such as $\beta$-lactamases like PER-1, PER-2, VEB1, AmpC cephalosporinases, carbapenemases like serine oxacillinases, metallo-b-lactamases, OXA-type carbapenemases and aminoglycoside-modifying enzymes (4).

The strains of bacteria producing these enzymes can clonally expand in the patient and transmit a particular type of resistance to other bacteria in the same or other bacterial 
species as well. Therefore, the procedures for the control of infections caused by these bacteria include the selection of an effective therapy for infected patients and the application of measures to control hospital infections and measures to control and rationally use antibiotics. In recent years, there is a positive trend in the development of resistant strains of bacteria in the outpatient population. In these patients, there is often a history of earlier hospitalization (surgery, intravascular or urinary catheter, stay in the intensive care unit and previous antibiotic therapy). However, some authors such as Bedenić et al. report of such isolates in-hospital patients who do not have any of the above risk factors (5).

\section{SUBJECTS AND METHODS}

This study observed 27 isolates of the gram-negative bacteria Pseudomonas aeruginosa and tested their resistance to various commonly used antibiotics. The strains were isolated from wounds of surgical patients of both sexes and different age groups. Patients were hospitalized in various departments (Department of Internal Medicine, Pediatrics, Dermatology, Ear, Nose and Throat, Neurology, Orthopedics, Oncology, and Physical and Rehabilitation medicine).

\section{RESULTS}

In $92 \%$ (25 patients) out of the total 27 samples, P. Aeruginosa was shown to have a resistance to imipenem and meropenem. $81 \%$ of the samples were resistant to ciprofloxacin, $78 \%$ to gentamicin, $67 \%$ to netilmicin, $55 \%$ to piperacillin, $48 \%$ to piperacillin/tazobactam ceftazidime, $41 \%$ to cefepime, $37 \%$ to aztreonam, $22 \%$ to tobramicin and $11 \%$ to amikacin. Resistance to colistin was not documented.

Therefore, according to the resistance patterns we see that the most effective antibiotic for PA infection in our sample population was colistin ( $100 \%$ sensitivity), followed by 
amikacin ( $88 \%$ sensitivity), tobramicin ( $81 \%$ sensitivity). The least effective antibiotics were found to be carbapenems ( $8 \%$ sensitivity) and ciprofloxacin (12\% sensitivity).

\section{DISCUSSION}

Pseudomonas aeruginosa is known to be a cause of skin and subcutaneous infections. Often these include post-operative infections in surgical wounds, burn victims and in chronic skin conditions $(6,7,8)$. According to certain liturature, PA is the 4th most common cause of surgical wound infections (9). One of the most worrisome characteristics of PA is its low antibiotic susceptibility, which is the result of the action of multidrug efflux pumps with chromosomally encoded antibiotic resistance genes such as mexAB and mexXY. Another mechanism of antibiotic resistance is the low permeability of the bacterial cellular envelopes (10). In addition to this intrinsic resistance, PA easily develops acquired resistance either chromosomally encoded gene mutations or by a horizontal gene transfer of antibiotic resistance determinants.

Hypermutation is another mechanism responsible for this bacteria's resistance to many commonly used antibiotics as it favours the selection of mutation-driven antibiotic resistance and results in chronic infections, whereas the clustering of several different antibiotic resistance genes in integrons favors the acquisition of antibiotic resistance determinants. The mechanisms underlying antibiotic resistance have been found to include the production of antibiotic-degrading or antibiotic-inactivating enzymes, outer membrane proteins to evict the antibiotics and mutations to change antibiotic targets.

The presence of antibiotic-degrading enzymes such as extended-spectrum $\beta$ lactamases such as PER-1, PER-2, VEB-1, AmpC cephalosporinases, carbapenemases like serine oxacillinases, metallo-b-lactamases, OXA-type carbapenemases, aminoglycosidemodifying enzymes have been reported. PA can also modify the targets of antibiotic action 
with one method including the methylation of $16 \mathrm{~S}$ rRNA to prevent aminoglycoside binding and the modification of DNA, or topoisomerase to protect it from quinolone action. It has also been reported to possess multidrug efflux pumps such as AdeABC and AdeDE efflux systems that confer resistance against number of antibiotic classes. An important factor found to be associated with antibiotic resistance is the decrease in the virulence capabilities of the resistant strain. Such findings have been reported in the case of rifampicin-resistant and colistin-resistant strains, in which a decrease in infective ability, quorum sensing and motility has been documented. Mutations in DNA gyrase are also commonly associated with PA antibiotic resistance and these mutations, when combined with others, confer a high resistance. Additionally, genes involved in cyclic-di-GMP signaling may contribute to resistance (4).

It has been seen that other bacterial strains have been shown to be developing resistance to many common antibiotics as well. Recent literature has also shown new evidence of drug resistance in other bacteria such as E. coli in which resistance to fluoroquinolones and cephalosporines is on the rise. The literature increasingly describes carbapenem resistant strains of Enterobacteriaceae as well (11-15). The resistance of Streptococcus pneumoniae to penicillin and other beta-lactams has been shown to be increasing worldwide.

The major mechanism of resistance in this bacteria involves the introduction of mutations in genes encoding penicillin-binding proteins (16). Acinetobacter is a gramnegative bacteria that causes pneumonia or bloodstream infections in critically ill patients. Multidrug-resistant Acinetobacter have become very resistant to antibiotics. Campylobacter causes diarrhea (often bloody), fever, and abdominal cramps. Serious complications such as temporary paralysis can also occur. Physicians rely on 
ciprofloxacin and azithromycin for treating patients with severe disease although Campylobacter is showing resistance to these antibiotics as well (17).

Antibiotics should only be used when needed as prescribed by health professionals (18). The prescriber should closely adhere to the five rights of drug administration: the right patient, the right drug, the right dose, the right route, and the right time (19). Narrowspectrum antibiotics are preferred over broad-spectrum antibiotics when possible, as effectively and accurately targeting specific organisms is less likely to cause resistnace (20). Cultures should be taken before treatment when indicated and the treatment potentially changed based on the susceptibility report $(21,22)$. For patients who take these medications without the supervision of a medical professional at home, education on proper use is imperative since non compliance is a major risk in the development of antibiotic resistant bacterial strains. Health care providers can also use a few simple steps to decrease the spread of multiresistant bacteria. These include proper and frequent handwashing and disinfecting between patients; and they should encourage the same to the patient, visitors, and family members (21)

\section{CONCLUSION}

The isolates of Pseudomonas aeruginosa show a high degree of resistance ( $>50 \%$ resistance) to commonly used antibiotics such as imipenem and meropenem, ciprofloxacin, gentamicin, netilmicin and piperacillin. It is therefore imperative that the physician take this into account when dealing with infections complicated with this bacteria. The results of this study may contribute to the creation of guidelines on empirical therapy in wound infection treatment in everyday practice. Further research is required and should be a continuation of this study. It should be our goal to prevent or slow down any bacterial resistance to new antibiotics used in 
the treatment of serious infections caused by multi-drug resistant Gram-negative bacteria. Wound infections caused by multi-drug resistant bacteria are a major therapeutic challenge for physicians and healthcare workers worldwide and should be dealt with appropriately in hopes to avoid further patient morbidity, decreasing hospital stay and ensuring the best care and management for the patient.

\section{AUTHORS' NOTE}

$\mathrm{Z}$ Veir conceived the reseach and paper, oversaw data collection, conducted data analysis, assisted in writing the manuscript and approved the final version. H Veir and M Franić both participated in the study design, data analysis and interpretation, critically revised the manuscript and approved the final version. $\mathrm{N}$ Beader participated in the data analysis and approved the final version. R Šalinović participated in the study design and critically revised the manuscript. The authors declare that they have no conflicts of interest. 


\section{REFERENCES}

1. Paterson DL. Recommendation for treatment of severe infections caused by Enterobacteriaceae producing extendede-spectrum beta lactamases (ESBLs). Clin Microbiol Infect 2000; 6: 460-3.

2. Bergogne-Berezin E, Towner KJ. Acinetobacter spp. as nosocomial pathogens: microbiological, clinical, and epidemiological features. Clin Microbiol Rev 1996; 9: $148-65$.

3. Vatopoulos AC, Philippon A, Tzouvelekis LS, Komninou Z, Legakis NJ. Prevalence of a transferable SHV-5 type beta-lactamase in clinical isolates of Klebsiella pneumoniae and Escherichia coli in Greece. J Antimicrob Chemother 1990; 26: 63548.

4. Wong, Alex; Rodrigue, Nicolas; Kassen, Rees. Genomics of Adaptation during Experimental Evolution of the Opportunistic Pathogen Pseudomonas aeruginosa. PLoS Genetics 1990; 8: e1002928.

5. Bedenić B, Vraneš J, Bošnjak Z, Marijan T, Mlinarić-Džepina A, Kukovec T, Anušić M, Beader N, Barl P, Leskovar V, Kalenić S. Emergence of CTX-M group 1 extended-spectrum $\beta$-lactamase-producing Klebsiella pneumoniae strains in the community. Med Glas 2010; 7: 32-9.

6. Vilar HE, Danel F, Livermore DM. Permeability to carbapenems of Proteus mirabilis mutants selected for resistance to imipenem of other $\beta$-lactams. J Antimicrob Chemother 1997; 40: 365-70.

7. Walsh FM, Amyes SGB. Microbiology and drug resistance mechanisms of fully resistant pathogens. Current opinion in microbiology 2004; 7: 439-44.

8. Walther-Rasmussen J, Hoiby N. Oxa-type carbapenemases. J Antimicrob Chemother 2006; 35: 373-83. 
9. $\mathrm{Yu}$ WL, Winokur P, Von Stein DL. First description of CTX-M- $\beta$-lactamases (CTXM-14 and CTX-M-3) in Taiwan. Antimicrob Agents. Chemother 2002; 46: 1098100.

10. Poole K. Efflux-mediated multiresistance in Gram-negative bacteria. Clinical microbiology and infection: the official publication of the European Society of Clinical Microbiology and Infectious Diseases 2002; 10: 12-26.

11. Dimou V, Dhanji H, Pike, R, Livermore, DM, Woodford N. Characterization of Enterobacteriaceae producing OXA-48-like carbapenemases in the UK. The Journal of antimicrobial chemotherapy 2012; 67: 1660-5.

12. Livermore DM, Canton R, Gnidakowski M, Nordmann P, Rossolini GM, Ariet G, Ayala J, Coque TM, Kern-Zdanowicz. CTX-M: changing tha face of ESBLs in Europe. J Antimicrob Chemother 2007; 59: 165-74.

13. Eisner A, Fagan EJ, Feierl G, Kessler HH, Marth E, Livermore DM, Woodford N. Emergence of Enterobacteriaceae isolates producing CTX-M extended-spectrum betalactamase in Austria. Antimicrob Agents Chemother 2006; 50: 785-7.

14. Mugnaioli C, Luzzaro F, De Luca F, Brigante G, Perilli M, Amicosante G, Stefani S, Toniolo A, Rossolini GM. CTX-M -type exteded -spectrum beta-lactamases in Italy: molecular epidemiology of an emerging countrywide problem. Antimicrob Agents Chemother 20006; 50: 2700-6.

15. Luzzaro F, Mezzatesta M, Mugnaioli C, Perilli M, Stefani S, Amicosante G, Rossolini GM, Toniolo A. Trends in production of extended-spectrum beta-lactamases among Enterobacteria of medical interest: report of the second Italian nationwide survey. J Clin Microbiol 2006; 44: 1659-64. 
16. Albrich WC, Monnet DL, Harbarth S; Monnet, DL; Harbarth S. Antibiotic selection pressure and resistance in Streptococcus pneumoniae and Streptococcus pyogenes. Emerging Infect. Dis. 2004; 10: 514-7.

17. Biggest Threats - Antibiotic/Antimicrobial Resistance CDC; www.cdc.gov.

18. The Five Rights of Medication Administration. www.ihi.org. 2015-10-30.

19. Swedish work on containment of antibiotic resistance - Tools, methods and experiences (PDF). Stockholm: Public Health Agency of Sweden. 2014; 16-17: 121128

20. NPS Medicinewise. National Prescribing Service Limited trading, Australia. http://www.nps.org.au; 2013.

21. CDC Features - Mission Critical: Preventing Antibiotic Resistance.www.cdc.gov. 2015.

22. Leekha S, Terrell CL, Edson RS. General Principles of Antimicrobial Therapy. Mayo Clinic Proceedings 2015; 86: 156-67. 